\title{
ANÁLISIS ESTRUCTURAL DEL SISTEMA TURÍSTICO RESIDENCIAL EN LA COSTA BLANCA
}

\author{
Antonio Aledo, Guadalupe Ortiz y Hugo García-Andreu \\ Departamento de Sociología \\ Universidad de Alicante
}

\section{RESUMEN}

Este artículo presenta una propuesta metodológica avanzada para el análisis sistémico del sector turístico residencial que permite identificar sus componentes y seleccionar los elementos claves que lo conforman. Se ha tomado como área de estudio la Costa Blanca (España). Para alcanzar este objetivo se ha empleado la herramienta informática MicMac $^{\oplus}$ (Matriz de Impactos Cruzados Multiplicación Aplicada a una Clasificación). El trabajo con esta herramienta a través de un panel de expertos ha permitido identificar ocho elementos claves del sistema turístico residencial en la Costa Blanca: Planificación y ordenación del territorio; Consumo de suelo; Precios; Prestigio del destino; Tipología constructiva; Inmigración de retirados; Paisaje, recursos naturales y playas; y Escena urbana. Esta metodología mejora la información en los procesos de toma de decisiones estratégicas en el planeamiento al identificar áreas de actuación prioritarias.

Palabras clave: análisis estructural, sistema turístico, Costa Blanca, turismo residencial.

\section{ABSTRACT}

This paper presents an advanced methodological proposal for the systemic analysis of the residential tourism sector, which allows the identification of its components and the selection of the key issues which comprise such system. It has been selected the Costa Blanca (Spain) as a case study for this research. With the aim of achieving this objective it has been used the software MicMac@ (Impact Matrix Cross-Reference Multiplication Applied to a Classification). The work with this tool by means of a Panel of Experts has led to the identification of eight key issues of the residential tourism system in the Costa Blanca: Territorial Planning; Land consumption; Prices; Prestige of the destination; Type of construction; Retirement migration; Landscape, natural resources and beaches; and Urban scenery. This methodology improves the information to be used in the strategic decision making processes for planning at a regional scale.

Key words: structural analysis, tourist system, Costa Blanca, residential tourism. 


\section{Introducción ${ }^{12}$}

La construcción de viviendas asociadas a la demanda de turismo residencial se ha convertido en las dos últimas décadas en la principal actividad económica de numerosas localidades del Mediterráneo español. El término turismo residencial pretende englobar una realidad compleja en la que las actividades turística y urbanística se hallan estrechamente unidas. Este concepto hace referencia a la demanda turística, así como la oferta de vivienda y de otros servicios, derivada tanto de los usuarios de segunda residencia con motivos fundamentalmente vacacionales como de las Migraciones Internacionales de Retirados, dados los muy similares efectos ${ }^{3}$ que a nivel local genera la actividad turística de ambos grupos (O’Really, 1995; Ferrer, Ribera y Reig, 1997; Rodríguez y Casado, 2000; Rodríguez y Warness, 2002; García, 2005; Aledo, García y Ortiz, 2006).

Algunas cifras pueden darnos una idea de la intensidad del fenómeno de la construcción. Entre 1998 y 2003 se edificaron en España el cuarenta por ciento del total de nuevas viviendas construidas en la UE (FCAC, 2003). Según el informe EXCELTUR (2005), entre 1991 y 2003 salieron al mercado 1.209.643 nuevas plazas de alojamiento extrahotelero en municipios del litoral de las comunidades del mediterráneo español y las Islas Canarias. El mismo informe calcula que entre las tres regiones españolas con mayores desarrollos turístico - residenciales - Comunidad Valenciana, Cataluña y Andalucía - suman un total de 3.689 .000 de viviendas de potencial uso turístico. Por lo que respecta a los procesos migratorios asociados a este fenómeno, en el año 2006, en las tres regiones mencionadas residían de forma oficial 204.169 personas nacidas en la Unión Europea mayores de 55 años (INE, 2006).

El crecimiento de este sector en la costa mediterránea española ha ocasionado una notable elevación de las rentas per capita de esos municipios, ha generado numerosos puestos de trabajo (García y Rodes, 2004) y ha ayudado a equilibrar las siempre deficitarias arcas municipales (Mazón, 2006). Los efectos positivos del turismo residencial quedan patentemente demostrados al observar que regiones que hasta hace poco eran generadoras de emigración se han convertido en focos de atracción de notables contingentes de inmigrantes laborales y residenciales (Monreal, 2001; Aledo, 2005).

No obstante, este crecimiento no está exento de contradicciones. Los efectos nocivos del turismo residencial en el Mediterráneo español han sido repetidamente señalados por una ya extensa bibliografía (Vera, 1992; Salvá, 1998; Torres, 2003; Mazón y Aledo, 2005), que hace referencia a sus impactos ambientales y sobre los recursos naturales (Gartner, 1987; Almenar, Bono y García, 2000; Olcina y Rico, 2005), a la falta de planificación, al déficit importantes de infraestructuras y servicios, a su fuerte concentración espacial y temporal (García y Rodes, 2004; Mazón y Aledo, 2005), a la dependencia de las haciendas municipales de los ingresos procedentes de las licencias de obras e IBI (Arrocha, 2005) y, por último,

1 Este artículo es resultado de una investigación financiada por Ministerio de Educación y Ciencia, a través del proyecto de I+D+i (2005-08) Turismo Residencial: análisis de la demanda y propuestas de reestructuración para destinos consolidados (SEJ 2005-04305)

2 Agradecemos la colaboración del Dr. Josep Ivars, del Insituto Universitario de Estudios Turísticos (Universidad de Alicante) con sus comentarios a este artículo.

3 Tanto los turistas vacacionales como los MIR comparten los mismos espacios turísticos, el mismo tipo de alojamiento y desarrollan actividades cotidianas similares dado que el tiempo de ocio es el elemento central de su vida cotidiana, aunque las actividades turísticas de los vacacionistas se consideran más activas que las de las MIR. 
a la corrupción urbanística que se extiende por demasiados municipios $\operatorname{costeros}^{4}$ (Gómez, Prieto y Strangeland, 2003; Vercher, 2004; Aledo, Mazón y Mantecón, 2007).

Para los municipios que han optado de forma casi monopolística por este tipo de modelo de desarrollo se dibuja, por tanto, un escenario de riesgo, ya sea por un futuro descenso de la demanda en este sector (Exceltur, 2005), por el consumo de su principal recurso, el suelo (Aledo, 2004), por la pérdida de calidad del producto (Bote, 1998), por la repercusión de los impactos ambientales que este sector produce (Vera, 1999), o por una combinación de todos estos factores.

Las respuestas que da el planeamiento español han resultado ineficaces debido a que implementan fórmulas caracterizadas por un enfoque sectorial, en el que prima lo local sobre lo regional, y con una fuerte tendencia a las actuaciones urbanísticas que dan lugar a espacios urbanos fragmentados, dispersos y desconectados entre sí. (Romero y Alberola, 2005).

Existe un consenso entre los especialistas (Vera, 1994, 2005; MUNRES, 1994; Antón, 1998; Romero et al., 2003) al señalar que estos déficit de carácter estructural ponen en peligro la capacidad de estos municipios para reorientar su modelo hacia fórmulas que sean económicamente viables a largo plazo, al mismo tiempo que cuestionan seriamente su sostenibilidad ambiental y reclaman un enfoque integral en las actuaciones urbanísticas en municipios turístico-residenciales.

Así pues, parece justificada la necesidad de reorientar el modelo de desarrollo en aquellos municipios que han optado por el turismo residencial como fórmula hegemónica de crecimiento económico. Las propuestas de reorientación se deben asentar sobre el fortalecimiento y mejora de los procesos de planeamiento local, para lo que se requiere de metodologías científicas que generen información de calidad.

En este sentido, este artículo presenta como principal objetivo la formulación y aplicación de una estrategia metodológica que facilite la producción de información útil y cualificada que se ponga al servicio de los encargados de la toma de decisiones en los procesos de planeamiento del desarrollo turístico residencial. Dada la multiplicidad de elementos y efectos que intervienen en el sector (Torres, 2003) se hace oportuno diseñar herramientas que simplifiquen la complejidad de su estructura. Con este fin, se ha optado por un enfoque sistémico-estructural, consistente en el análisis de las partes componentes de un sistema - en este caso, el sistema turístico-residencial-y de sus relaciones recíprocas. Este enfoque permite la organización de información compleja, a la vez que proporciona una aproximación integral al objeto de estudio.

En este artículo se expondrá, en primer lugar, la metodología empleada para el análisis del sistema turístico-residencial a escala territorial, en este caso de la Costa Blanca, en el sudeste español. Seguidamente, se presentarán algunos de los usos analíticos que se le pueden dar a esta herramienta para enfoques integrales en el planeamiento de municipios turístico-residenciales.

\section{Metodología: los enfoques sistémicos y estructurales aplicados al análisis del turismo residencial}

Aunque no existe experiencia previa de análisis estructurales de este modelo específico de desarrollo, sí existen algunos análisis del sistema turístico en su globalidad. Como

4 España, octava potencia económica del mundo, se sitúa en el puesto 23, de 159 países, en el Índice de Percepción de la Corrupción de 2005 elaborado por la organización Transparencia Internacional (en Greenpeace, 2006). 
señala Ivars (2003), muchas de las propuestas de carácter metodológico en materia de planificación turística conciben el turismo como un sistema (Boullon, 1985; Gunn, 1994, 1997; Baud-Bovy y Lawson, 1998 y Hall, 2000). En este sentido, se ha aplicado la Teoría General de Sistemas a los estudios turísticos, adoptando tanto un enfoque de análisis genérico de la actividad, como uno más específico de gestión de recursos o de organización de empresas turísticas (Mill y Morrison, 1992, Mathieson y Wall, 1992).

Numerosos estudios emplean enfoques parciales del sistema turístico, tales como los que Clary (1993) denomina enfoques estructurales (referidos a las formas de ocupación del espacio turístico: infraestructuras, oferta de alojamiento, etc.) y enfoques funcionales (referidos a los flujos de población, dinero, información, etc., propios de la actividad turística) (Ivars, 2003). Otros autores como Leiper (1995) identifican el sistema turístico desde una perspectiva del viaje (origen, viaje y destino), o con los aspectos económicos de la actividad turística (oferta, demanda, marketing, etc.) (Mill y Morrison, 1992).

Pero el sistema turístico residencial, dadas sus características específicas, no puede ser entendido de forma segmentada, enfocando su análisis únicamente desde la perspectiva de la oferta, de la demanda o de flujos de población. Los efectos ambientales, económicos, sociales, culturales y políticos que este modelo de desarrollo tiene a escala local y regional, así como las estructuras institucionales y sociales que favorecen su implantación, entre otras muchas cuestiones, no pueden quedar fuera a la hora de analizar este sistema.

El reto de delinear el sistema del turismo residencial y sus efectos sobre las comunidades y su territorio se enfrenta, por tanto, a la falta de análisis sistémicos y estructurales previos. Dos razones explican este déficit. En primer lugar, la mayoría de las investigaciones sobre turismo residencial se caracterizan por un enfoque sectorial, focalizando el análisis en aspectos bien ambientales (Gartner, 1987), demográficos (Casado, 2000), económicos (Exceltur, 2005) o socioculturales (García, 2005). Y en segundo lugar, la ambigua naturaleza del sector, que se sitúa entre las actividades urbanísticas y las dinámicas turísticas, introduce una mayor indefinición de los límites del sistema (Hall y Müller, 2004; Mazón, 2006).

Siendo conscientes de las especificidades del turismo residencial respecto de la actividad turística tradicional, se diseñó y se testó una metodología sistémico-estructural adaptada a las características concretas de este sector turístico. Como paso previo fue necesario acotar un marco espacial que pusiera límites al dibujo del sistema. El espacio seleccionado fue la Costa Blanca, que corresponde a los municipios turísticos costeros de la provincia de Alicante (España). Las razones de esta selección fueron, por un lado, la fuerte presencia del sector turístico-residencial en esta región, así como la información que este grupo investigador posee sobre este territorio. Una vez delimitada el área de estudio, se procedió a la identificación de los múltiples elementos que componen el sistema turístico residencial en la Costa Blanca, así como al análisis de tales componentes y las interacciones que establecen entre sí. Finalmente, esta información permitió determinar las áreas de actuación prioritaria o elementos clave del sistema. Para llevar a cabo estas últimas tareas, se optó por la conformación de un Panel de Expertos en materia turístico-residencial. El trabajo de especialistas en las distintas áreas relacionadas con este modelo turístico, aseguró la producción de información completa y de calidad.

A continuación, describiremos con mayor detalle los pasos seguidos en esta investigación. 


\subsection{Primera fase: Identificación de los componentes del sistema turístico-residencial de la Costa Blanca mediante un Panel de Expertos}

La primera fase de esta investigación perseguía la identificación del conjunto de elementos o variables que componen el sistema del turismo residencial. Para ello, un total de quince expertos en diversas disciplinas relacionadas con el campo del turismo (geografía, economía, derecho, antropología, sociología, turismo, promoción inmobiliaria y asociaciones cívicas) y procedentes tanto del mundo académico, como de la administración pública y la empresa privada, fueron convocados a formar parte de un Panel de Expertos en Turismo Residencial en la Costa Blanca.

La primera tarea que llevó a cabo este Panel de Expertos fue cumplimentar un cuestionario abierto mediante el cual se les solicitó que identificaran las variables o factores determinantes del modelo actual de turismo residencial; esto es, aquellas variables que componen, definen, influyen o explican el sistema del turismo residencial en la Costa Blanca. Se trazó, de este modo, un mapa desordenado de los componentes del sistema que serviría como base del trabajo posterior.

Para facilitar la reflexión acerca de un objeto de estudio tan amplio, se indicaron seis grandes áreas en las que el turismo residencial puede manifestarse: Medio Ambiente, Economía, Política, Normativa, Factores Socioculturales y Urbanismo. Para asegurar una correcta comprensión de los factores propuestos por los expertos, se les pidió que proporcionasen una definición o explicación de cada uno.

Las respuestas del Panel de Expertos a este sencillo cuestionario permitieron obtener un listado de un total de ochenta y cinco variables, con sus respectivas definiciones que, según los expertos, determinan o explican el sistema turístico-residencial ${ }^{5}$. Dado que el trabajo con el listado completo de variables habría sido excesivamente complicado, era necesario priorizar las variables identificadas. Para ello, se pidió al grupo de expertos que puntuaran de 0 a 10 cada una de las variables en función de su importancia o protagonismo en las características y funcionamiento actuales, así como en la evolución futura del turismo residencial en la provincia de Alicante. De este modo, se obtuvo un listado ordenado de los ochenta y cinco elementos (Tabla 1), del cual se seleccionaron las veinticuatro variables principales $^{6}$, que serían la base para el trabajo en la siguiente fase de la investigación.

\subsection{Segunda fase: análisis del funcionamiento del sistema turístico-residencial}

Para abordar la segunda etapa de la investigación, se reunió al Panel de Expertos en una jornada de trabajo con el objetivo de analizar las relaciones que mantienen las variables dentro del sistema turístico-residencial. Se optó por el empleo de la herramienta informática $\mathrm{MicMac}^{\odot 7}$, que permite visibilizar las interrelaciones existentes entre los distintos componentes de un sistema, identificando las relaciones de dependencia e influencia que

5 Un planteamiento similar, ha sido elaborado por J.Perles (2006) que, empleando la Teoría de la Ventaja Competitiva de Michael Porter (1990), ha construido un cluster de Turismo Residencial compuesto por siete elementos: Motivaciones, Hospedaje, Alimentación, Agencias de Viaje, Atracciones, Capacitación y Servicios de Apoyo.

6 Del listado de ochenta y cinco variables se seleccionaron las veinticuatro primeras, ya que un número mayor habría sido poco manejable para un Panel de Expertos como el que se conformó, debido al sistema de cruces entre variables que requería la técnica utilizada.

7 MicMac (Matriz de Impactos Cruzados Multiplicación Aplicada a una Clasificación) ${ }^{\circledR}$, es una herramienta desarrollada por LIPSOR (Laboratoire d'Investigation en Prospective, Stratégie et Organisation), dirigido por el profesor Michel Godet. 
Tabla 1

ELEMENTOS COMPONENTES DEL SISTEMA TURÍSTICO-RESIDENCIAL SEGÚN UN PANEL DE EXPERTOS, ORDENADOS EN FUNCIÓN DE SU IMPORTANCIA O PROTAGONISMO

\begin{tabular}{|c|c|c|c|}
\hline Orden & Variable & Orden & Variable \\
\hline 1 & Clima & 44 & Directrices Supranacionales \\
\hline 2 & Paisaje, playas y recursos naturales & 45 & Legislación hídrica nacional \\
\hline 3 & Servicios españoles & 46 & Calidad del agua \\
\hline 4 & Seguridad ciudadana & 47 & Legislación de costas nacional \\
\hline 5 & Inmigración de retirados & 48 & Legilación autonómica sobre campos de golf \\
\hline 6 & Precios & 49 & Prestaciones por jubilación \\
\hline 7 & Oferta de agua & 50 & Liberalización de mercado del suelo \\
\hline 8 & Búsqueda de calidad de vida & 51 & Importancia otorgada al ocio \\
\hline 9 & Prestigio del destino & 52 & Estacionalidad \\
\hline 10 & Calidad de oferta de alojamiento turístico & 53 & residuos \\
\hline 11 & Planificación y ordenación del territorio & 54 & Ingresos municipales \\
\hline 12 & Demanda de agua & 55 & Envejecimiento de la estructura poblacional local \\
\hline 13 & Legislación urbanística autonómica & 56 & gasto municipal \\
\hline 14 & Seguridad jurídica & 57 & Legislación ambiental nacional \\
\hline 15 & Consumo de suelo & 58 & Tipos de interés \\
\hline 16 & Paz / estabilidad social & 59 & Hábitos de consumo turistas-residentes \\
\hline 17 & Estilo de vida local/ «cultura mediterránea» & 60 & Dinamización economía local \\
\hline 18 & Densidad del tráfico & 61 & Organización del tiempo y espacio de trabajo \\
\hline 19 & Valores ambientalistas turistas-residentes & 62 & Procesos administrativos de producción de suelo \\
\hline 20 & Evaluaciones de impacto ambiental & 63 & Flexibilidad de la legislación inmobiliaria \\
\hline 21 & Tipología constructiva & 64 & Integración entre comunidades local-extranjera \\
\hline 22 & Escena urbana & 65 & Diversidad cultural \\
\hline 23 & Estructura de alojamiento turístico & 66 & Producción de lugares sin identidad \\
\hline 24 & Corrupción & 67 & Nivel De Cooperación Administrativa \\
\hline 25 & Infraestructuras de transporte & 68 & Legislación turística autonómica \\
\hline 26 & Protagonismo del ayuntamiento en la planif. Territorial & 69 & Valores individualistas \\
\hline 27 & Valores ambientalistas locales & 70 & Tratamiento fiscal de la vivienda \\
\hline 28 & Integración económica europea & 71 & Crisis de la agricultura \\
\hline 29 & Situación económica en países emisores & 72 & Grupos de presión económicos \\
\hline 30 & Oferta complementaria (no alojamiento y restauración) & 73 & Legislación urbanística nacional \\
\hline 31 & Densidad demográfica & 74 & Nuevas tecnologías de comunicación \\
\hline 32 & Reconversión residencial de la vivienda rural dispersa & 75 & Estructura de sectores productivos \\
\hline 33 & Legislación ambiental autonómica & 76 & $\begin{array}{l}\text { Nivel de conocimiento sobre procedimientos } \\
\text { administrativos de extranjeros }\end{array}$ \\
\hline 34 & Idioma & 77 & riesgos naturales \\
\hline 35 & Integración en la unión europea & 78 & Nivel de rentabilidad de otras inversiones \\
\hline 36 & Modalidad de asentamiento & 79 & Capacidad de endeudamiento de la familia española \\
\hline 37 & Demanda energética & 80 & Participación política de extranjeros. \\
\hline 38 & Nivel de vida & 81 & Inmigración laboral \\
\hline 39 & Legislación de calidad de la edificación & 82 & mercado de trabajo. \\
\hline 40 & Colonias de extranjeros & 83 & Transfuguismo político \\
\hline 41 & Contaminación atmosférica & 84 & Facilidades locales a la inversión inmobiliaria \\
\hline 42 & Participación ciudadana y asociacionismo proactivo & 85 & Legislación turística nacional \\
\hline 43 & Relación del extranjero con la administración & & \\
\hline
\end{tabular}

Elaboración propia. 
se establecen entre ellos (Godet, 1991, 2000). El conocimiento del nivel de dependencia o de influencia que una variable tiene respecto al resto de componentes del sistema puede ser útil para mejorar las acciones de planeamiento y diseño estratégico. En este sentido, resulta relevante conocer qué variables ejercen una influencia mayor sobre el conjunto del sistema, puesto que la actuación sobre ellas tendrá efectos sobre múltiples factores. Asimismo, es conveniente saber qué elementos son más sensibles o vulnerables ante cambios producidos en otras variables.

Para identificar las relaciones de influencia y dependencia entre las veinticuatro variables principales escogidas en la fase previa, los expertos completaron, durante la jornada de trabajo, una matriz de doble entrada en la que las variables aparecían en las filas y columnas. Se les pidió, para ello, que puntuaran, de cero a tres, el nivel de influencia de las variables situadas en las filas de la matriz sobre el resto de variables o columnas, dónde: $0=$ no existe influencia; 1 = influencia moderada; $2=$ influencia media; y $3=$ influencia fuerte. De este modo, la suma de las puntuaciones de cada fila de la matriz ofrece un indicador del nivel de influencia de las variables, puesto que suma el grado en que esa variable (fila) influye sobre el resto de variables de la matriz. Igualmente, la suma de las puntaciones de cada columna indica el nivel de dependencia de las variables, puesto que indica el grado en que esa variable (columna) es influida por el resto de variables de la matriz (Ver Tabla 2).

Tabla 2

VISTA DE LA MATRIZ DE RELACIONES DE LAS 24 VARIABLES PRINCIPALES

\begin{tabular}{|c|c|c|c|c|c|c|c|c|c|c|c|c|c|c|c|c|c|c|c|c|c|c|c|c|}
\hline & &  & 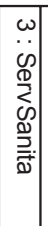 & 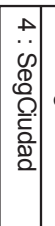 & & & 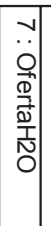 & 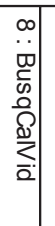 & 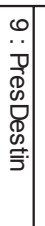 & 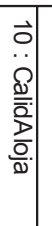 & 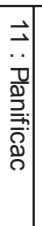 & 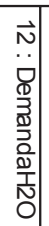 & 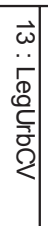 & 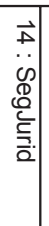 & 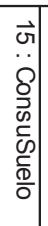 & & 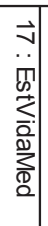 & & $\begin{array}{l}\overrightarrow{0} \\
2 \\
\overrightarrow{0} \\
\frac{0}{0} \\
\overline{0} \\
0 \\
\vec{\lambda}\end{array}$ & & $\begin{array}{c}N \\
\ddot{n} \\
\ddot{7} \\
\overline{0} \\
\delta \\
0 \\
\stackrel{0}{7}\end{array}$ & 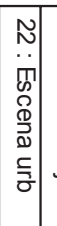 & 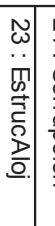 & 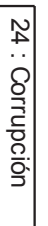 \\
\hline Clime & $\underline{0}$ & 3 & 0 & 0 & 3 & 0 & 3 & 3 & 3 & 1 & 1 & 2 & 0 & 0 & \begin{tabular}{|l|}
1 \\
\end{tabular} & 0 & 2 & 0 & 0 & \begin{tabular}{|l|l}
0 & 2 \\
\end{tabular} & 2 & \begin{tabular}{|l|}
2 \\
\end{tabular} & \begin{tabular}{l|l}
0 & 0 \\
\end{tabular} & 0 \\
\hline 2: Pais & & 0 & 0 & 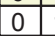 & 1 & 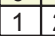 & 2 & 2 & 2 & 1 & 2 & 0 & 0 & & 2 & & \begin{tabular}{|l|}
0 \\
\end{tabular} & 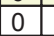 & \begin{tabular}{|l|}
1 \\
\end{tabular} & $\frac{1}{1}$ & \begin{tabular}{|l|}
3 \\
\end{tabular} & 2 & \begin{tabular}{l|l}
0 & 0 \\
0
\end{tabular} & 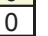 \\
\hline $3:$ Ser & & $\underline{0}$ & 0 & 0 & 3 & 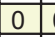 & 0 & 3 & $\leq$ & 0 & 0 & & 0 & & 0 & & 0 & 7 & 0 & 0 & 0 & 0 & \begin{tabular}{l|l}
0 & 0 \\
\end{tabular} & 0 \\
\hline $4:$ Seg & & 0 & 0 & 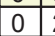 & 2 & 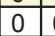 & 0 & 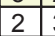 & 3 & 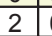 & 0 & c & $\begin{array}{ll}0 \\
\end{array}$ & & 0 & & \begin{tabular}{|l|}
1 \\
\end{tabular} & & 0 & & 0 & 4 & 0 & 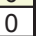 \\
\hline $5: \ln m i$ & c & 0 & 3 & 1 & $\overline{0}$ & 2 & 0 & 0 & 1 & 0 & 1 & 3 & 0 & ( & 3 & 0 & 0 & 0 & 1 & 0 & 3 & 1 & \begin{tabular}{l|l}
2 & 0 \\
2
\end{tabular} & 0 \\
\hline 6:Prec & 0 & 0 & 4 & 8 & 3 & 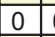 & 0 & 3 & 2 & 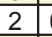 & $\therefore$ & & 0 & & 3 & & 2 & & 0 & 0 & 3 & 1 & 3 & 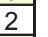 \\
\hline $7:$ Ofer & & 2 & 0 & 0 & 0 & 0 & 0 & 0 & 0 & 0 & 2 & & 2 & & \begin{tabular}{|l|}
1 \\
\end{tabular} & & 2 & 0 & 1 & 2 & 1 & 2 & 1 & 0 \\
\hline $8:$ Busq & 0 & $\frac{2}{2}$ & 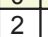 & 0 & 3 & 0 & 0 & 0 & 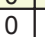 & 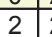 & 2 & & \begin{tabular}{|l} 
\\
0
\end{tabular} & & 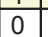 & & $\frac{2}{2}$ & 7 & $\begin{array}{l} \\
0\end{array}$ & 0 & 1 & $\frac{1}{1}$ & c & 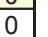 \\
\hline 9: Pres & 0 & 0 & 2 & 1 & 3 & 3 & 0 & 3 & 0 & 2 & 0 & 1 & 0 & 0 & 2 & & 2 & 1 & \begin{tabular}{|l|}
1 \\
\end{tabular} & 0 & 3 & 2 & 2 & 1 \\
\hline $10: \mathrm{Ca}$ & ( & 2 & $\begin{array}{ll}0 \\
\end{array}$ & 0 & 4 & 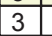 & 0 & 1 & 2 & 0 & 0 & & 0 & & \begin{tabular}{|l}
0 \\
\end{tabular} & & \begin{tabular}{|l}
0 \\
\end{tabular} & 7 & 0 & 7 & \begin{tabular}{|l|}
0 \\
\end{tabular} & 3 & c & 0 \\
\hline 11: Pla & 0 & 3 & \begin{tabular}{|l|}
1 \\
\end{tabular} & 2 & 0 & 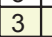 & 0 & 1 & 2 & 0 & 0 & 3 & 0 & 7 & \begin{tabular}{|l|}
3 \\
\end{tabular} & 0 & 0 & 3 & 0 & $\begin{array}{l}1 \\
\end{array}$ & 3 & 3 & \begin{tabular}{l|l}
3 & 3 \\
\end{tabular} & 3 \\
\hline $12: \mathrm{De}$ & & 0 & 0 & 1 & 0 & 2 & 3 & 0 & 0 & 0 & 2 & & 2 & & 0 & & 0 & \begin{tabular}{|l|}
0 \\
\end{tabular} & 0 & 0 & \begin{tabular}{|l|}
1 \\
\end{tabular} & 0 & 1 & 1 \\
\hline 13: LegUrbCV & $\underline{0}$ & 3 & 1 & 0 & 3 &  & 0 & 更 & 2 & 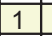 & 3 & 1 & 0 & 3 & 3 & 0 & 0 & 1 & 1 & 2 & 3 & 3 & \begin{tabular}{l|l}
1 & 3 \\
\end{tabular} & 3 \\
\hline $14:$ Seg & & 0 & 0 & 0 & 2 & 0 & 8 & 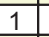 & 3 & 0 & . & $\mathrm{c}$ & 2 & $\mathrm{c}$ & 0 & 0 & 8 & 0 & 8 & 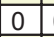 & 0 & 0 & 0 & 1 \\
\hline $15: \mathrm{Cor}$ & 0 & 3 & 1 & 1 & 0 &  & 2 & 0 & 1 & 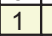 & 2 & & 3 & & 0 & & 0 & 2 & 2 & 1 & 2 & 1 & 2 & 3 \\
\hline $16:$ & & 0 & 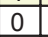 & 1 & 2 &  & 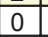 & 2 & 4 & 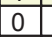 & 0 & 0 & \begin{tabular}{|l|}
1 \\
\end{tabular} & & $\begin{array}{l} \\
\end{array}$ & & 4 & 0 & $\mid$ & 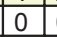 & \begin{tabular}{|l|l}
0 \\
\end{tabular} & & & \\
\hline $17:$ Est & 0 & 0 & 0 & 0 & 2 & 0 & 0 & 3 & 1 & 0 & 0 & c & 0 & 0 & 0 & 0 & 0 & 0 & 0 & 0 & 1 & 2 & 0 & 0 \\
\hline $18:$ & & 1 & 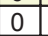 & 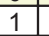 & 0 & 0 & 0 & 1 & 4 & 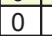 & 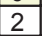 & 0 &  & 0 & 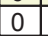 & 0 &  & 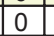 & 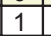 &  & 4 & 2 & & \\
\hline $19: \mathrm{Va}$ & $\mathrm{c}$ & $\underline{0}$ & 0 & 0 & 0 & 0 & 0 & 1 & 2 & $\underline{0}$ & 2 & 1 & 2 & 0 & 0 & & 0 & & 0 & 0 & 0 & & 0 & 0 \\
\hline $20: \mathrm{EIA}$ & 0 & 1 & 0 & 0 & 0 & 0 & 0 & 0 & 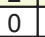 & 0 & 1 & 0 & \begin{tabular}{|l|l}
0 \\
\end{tabular} & 0 & 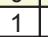 & 0 & 0 & 0 & 0 & 0 & 0 & 1 & \begin{tabular}{l|l}
0 & 0 \\
\end{tabular} & 0 \\
\hline nstr & c & 2 & 0 & 0 & 2 & 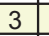 & 0 & 2 & 3 & 2 & 4 & 3 & 0 & 0 & 3 & 0 & 2 & 2 & 1 & 0 & 0 & 2 & 1 & 0 \\
\hline $22:$ Es & 0 & 3 & 0 & 0 & 1 & 2 & 0 & 3 & 3 & \begin{tabular}{|l}
3 \\
\end{tabular} & 0 & 1 & 0 & 0 & 0 & 0 & \begin{tabular}{|l|}
2 \\
\end{tabular} & 2 & 2 & 0 & 0 & 0 & \begin{tabular}{l|l}
0 \\
0
\end{tabular} & 0 \\
\hline $3: 1$ & & 0 & 0 & 4 & & 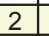 & 5 & 0 & & 0 & & 3 & 0 & 0 & 3 & 0 & $\begin{array}{l}0 \\
\end{array}$ & 3 & 1 & 0 & 3 & 2 & 0 & 5 \\
\hline $24:$ Cor & 0 & 2 & 0 & 1 & 0 & 3 & 2 & 0 & 2 & \begin{tabular}{|l|}
0 \\
\end{tabular} & 3 & 0 & 2 & 1 & \begin{tabular}{|l|}
3 \\
\end{tabular} & 2 & 0 & $\begin{array}{l} \\
\end{array}$ & 0 & 2 & 2 & 0 & 0 & 0 \\
\hline
\end{tabular}


La elaboración de esta matriz ofrece desde el primer momento una información muy valiosa sobre la situación de cada variable dentro del sistema. Podemos a partir de ella averiguar qué interrelaciones establece un determinado elemento del sistema con el resto, permitiendo de esta manera identificar sobre qué otros elementos podemos actuar para ejercer un efecto sobre la variable elegida. Por ejemplo, si una corporación municipal estuviese interesada en mejorar la calidad de la oferta de alojamiento turístico local, debería proceder a identificar qué otros elementos del sistema ejercen influencia sobre esta variable. De esta manera, la matriz indicaría qué elementos del sistema turístico local influirían sobre esta variable y, por lo tanto, quedarían identificadas las áreas concretas de actuación indirecta para conseguir el objetivo final. En este caso, se puede observar cómo, para introducir cambios en la calidad del alojamiento turístico de un municipio, podrían diseñarse estrategias de actuación indirecta sobre esta variable. Sería posible plantearse, por ejemplo, acciones de mejora de la escena urbana y del paisaje, plantear modificaciones en la legislación urbanística autonómica, en la tipología constructiva o en los patrones de consumo de suelo, o bien, considerar la calidad del alojamiento como una variable dependiente del prestigio del destino turístico.

Las posibilidades de uso que ofrece esta herramienta no se limitan a este acercamiento exploratorio, sino que permite elaboraciones analíticas más complejas. El primer análisis que proporciona el $\mathrm{MicMac}^{\odot}$, genera una clasificación de las variables según su nivel de influencia y su nivel de dependencia. El nivel de influencia para cada variable se obtiene de la suma de las puntuaciones de cada fila (Tabla 2). Por su parte, el nivel de dependencia para cada variable se consigue mediante el sumatorio de las puntuaciones de su columna. La clasificación del nivel de influencia permite identificar aquellas variables que ejercen un mayor efecto sobre el resto y, por lo tanto, señala posibles vías de actuación indirecta sobre otras variables. La clasificación por nivel de dependencia muestra qué variables son más susceptibles de ser afectadas por el cambio en otros componentes del sistema. Por ejemplo, la Tabla 3 muestra que la actuación sobre la Legislación Urbanística Autonómica ejercería un impacto significativo sobre el resto de variables del sistema. Por otro lado, el prestigio del destino aparece como la variable más vulnerable ante alteraciones en el sistema, puesto que recibe mucha influencia por parte de otras variables.

El proceso de toma de decisiones durante el planeamiento exige priorizar las áreas de actuación. Una forma de identificar áreas prioritarias de actuación puede ser distinguir dentro del sistema aquellas variables que al mismo tiempo son las más influyentes y más dependientes. Se puede considerar estas variables como las variables clave del sistema (Godet, 2000), puesto que su comportamiento tendrá un gran efecto sobre el del conjunto del sistema y son a su vez vulnerables ante los cambios de otras variables y, por lo tanto, son también susceptibles de ser modificadas. No sería adecuado definir como variables clave a aquéllas que tienen un nivel de influencia alto pero cuyo nivel de dependencia es bajo, como es el caso de la variable Clima, puesto que las posibilidades de ejercer un efecto sobre esta variable son muy pequeñas. Del mismo modo, no sería conveniente definir como variables clave aquellas con un alto nivel de dependencia pero bajo nivel de influencia puesto que las acciones ejercidas sobre ellas no tendrían efectos significativos sobre el resto del sistema.

El MicMac permite la identificación de estas variables clave a partir de la combinación de las puntuaciones obtenidas en dependencia e influencia. El programa ofrece un eje de coordenadas, en cuyo cuadrante superior derecho se ubican aquéllas variables que son muy dependientes y muy influyentes al mismo tiempo (Gráfico 1). 
Tabla 3

COMPARACIÓN DE LAS CLASIFICACIONES DE VARIABLES COMPONENTES DEL SISTEMA TURÍSTICO-RESIDENCIAL SEGÚN NIVEL DE INFLUENCIA Y

\section{DEPENDENCIA}

\begin{tabular}{|c|c|c|c|}
\hline \multicolumn{2}{|r|}{ CLASIFICACIÓN SEGÚN NIVEL DE INFLUENCIA } & \multicolumn{2}{|r|}{ CLASIFICACIÓN SEGÚN NIVEL DE DEPENDENCIA } \\
\hline 1 & Legislación urbanística autonómica & 1 & Prestigio del destino \\
\hline 2 & Planificación y ordenación del territorio & 2 & Búsqueda de calidad de vida \\
\hline 3 & Consumo de suelo & 3 & Inmigración de retirados \\
\hline 4 & Prestigio del destino & 4 & Tipología constructive \\
\hline 5 & Tipología constructiva & 5 & Escena urbana \\
\hline 6 & Clima & 6 & Precios \\
\hline 7 & Precios & 7 & Consumo de suelo \\
\hline 8 & Corrupción & 8 & Paisaje, recursos naturales y playas \\
\hline 9 & Escena urbana & 9 & Demanda de agua \\
\hline 10 & Paisaje, recursos naturales y playas & 10 & Planificación y ordenación del territorio \\
\hline 11 & Inmigración de retirados & 11 & Calidad de la oferta de alojamiento turístico \\
\hline 12 & Oferta de agua & 12 & Estructura del alojamiento turístico \\
\hline 13 & Estructura del alojamiento turístico & 13 & Densidad del tráfico \\
\hline 14 & Búsqueda de calidad de vida & 14 & Estilo de vida mediterráneo \\
\hline 15 & Calidad de la oferta de alojamiento turístico & 15 & Corrupción \\
\hline 16 & Demanda de agua & 16 & Legislación urbanística autonómica \\
\hline 17 & Densidad del tráfico & 17 & Oferta de agua \\
\hline 18 & Seguridad ciudadana & 18 & Valores ambientalistas de los turistas-residentes \\
\hline 19 & Estabilidad política y social & 19 & Servicios sanitarios \\
\hline 20 & Seguridad jurídica & 20 & Evaluación de impacto ambiental \\
\hline 21 & Estilo de vida mediterráneo & 21 & Seguridad ciudadana \\
\hline 22 & Valores ambientalistas de los turistas-residentes & 22 & Seguridad juridical \\
\hline 23 & Servicios sanitarios & 23 & Estabilidad política y social \\
\hline 24 & Evaluación de impacto ambiental & 24 & Clima \\
\hline
\end{tabular}

Elaboración propia.

Una vez identificadas las ocho variables clave del sistema turístico-residencial de la Costa Blanca, se debe analizar el conjunto de relaciones que estos elementos clave mantienen entre sí y con el resto de las veinticuatro variables principales. Esta tarea tiene como objetivo prever las consecuencias que tendría sobre el conjunto del sistema las actuaciones sobre las variables clave. Esto permitiría al tomador de decisiones maximizar el impacto positivo de sus acciones y minimizar sus posibles efectos negativos.

Previamente a este análisis hay que dotar de contenido contextual a cada una de las variables clave, a fin de interpretar de manera adecuada la función que desempeña, el sentido de las relaciones que mantiene con el resto y estimar los efectos que puede provocar su modificación sobre el resto de las variables. 


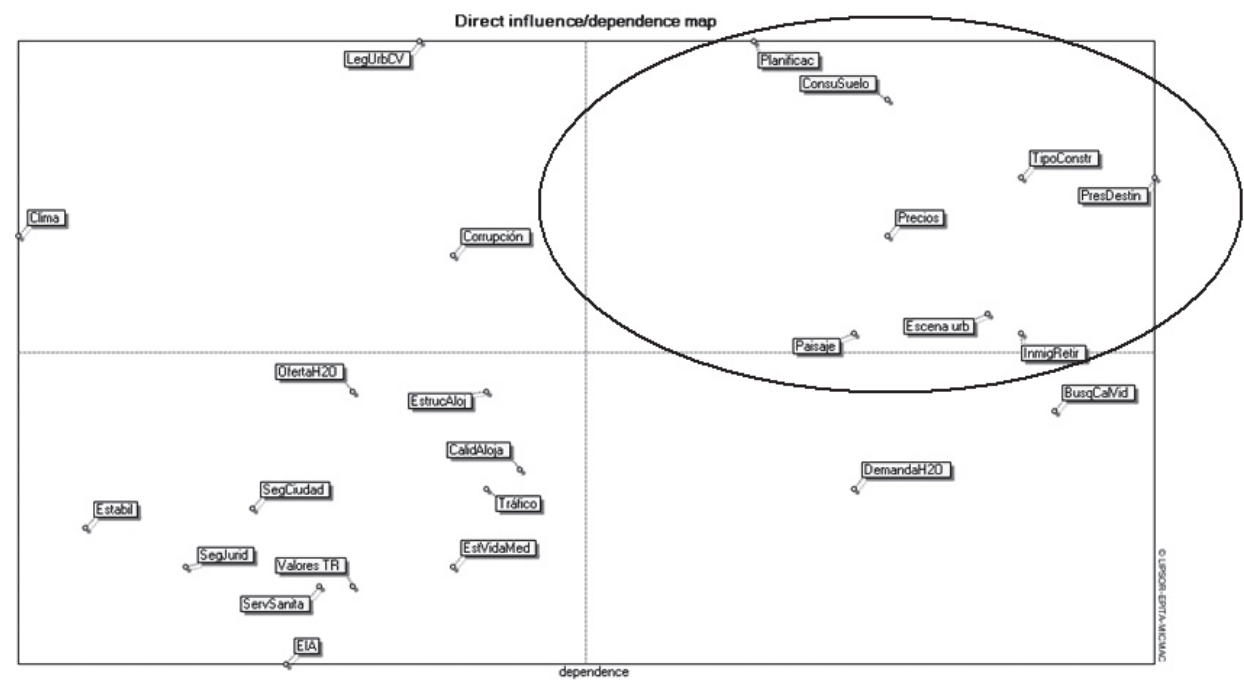

GRÁfico 1. Mapa de influencia / dependencia de las variables componentes del sistema turísticoresidencial de la provincia de Alicante

Elaboración propia.

\subsection{Contextualización de las variables clave}

Se ofrece a continuación un análisis de las ocho variables que han aparecido como elementos clave del sistema. Se presenta, para cada una de ellas, la definición que se consensuó previamente por los expertos, en función de la cual se trabajó durante las reuniones del Panel de Expertos, así como una contextualización de la variable en el marco socioterritorial del turismo residencial en la Costa Blanca.

\subsubsection{Planificación y ordenación del territorio}

Definición: regulación, a través de diversos instrumentos de planeamiento, del uso y desarrollo del suelo y de las comunicaciones de un municipio.

Contextualización: La bibliografía académica que ha estudiado el desarrollo del turismo residencial en la Costa Blanca concuerda en apuntar la falta de planeamiento a nivel urbano y de planes de ordenación territorial a nivel supramunicipal como uno de los principales elementos que explican el mal desarrollo del modelo (Vera, 1987; Navalón, 1995; MUNRES, 1995; Costa y Jiménez, 1999; Mazón, 2006). En España, son los Planes Generales de Ordenación Urbana (PGOU) los encargados de planificar los desarrollos y crecimientos urbanos. Sin embargo, en numerosos municipios estos planes han carecido de implementación y se han sustituido por acciones parciales (Greenpeace, 2006) que han contribuido a construir ciudades fragmentadas, inconexas, con un grave déficit en infraestructuras y servicios y, en definitiva, sin un modelo de ciudad previamente definido — que es lo que proporciona los PGOU (Aledo y Mazón, 2005). 


\subsubsection{Consumo de suelo}

Definición: utilización de extensiones de territorio municipal para el desarrollo del turismo residencial.

Contextualización: La expansión espacial del turismo residencial ha ocasionado un elevado consumo de suelo ligado a intensos procesos de cambio de uso del suelo de agrícola a urbano (Vera, 1992; Peña et al 2007). En una primera etapa, se ocupó la línea de costa para, posteriormente, comenzar la construcción de urbanizaciones en el interior. La alta demanda de viviendas turísticas por parte del mercado nacional y europeo ha estimulado este fuerte proceso constructivo en todo el Mediterráneo español (Almenar, Bono y García, 2000). El resultado ha sido altas tasas de masificación en los municipios de costa (OSE, 2006a) y la traslación de impactos socioambientales a los municipios de interior.

\subsubsection{Prestigio de destino}

Definición: percepción de la calidad de un destino turístico en las zonas emisoras de turistas-residentes.

Contextualización: El producto residencial engloba no sólo el conjunto residencial - compuesto por vivienda y urbanización - sino también el conjunto de servicios e infraestructuras, paisaje y recursos naturales, así como los recursos humanos, estilo de vida y recursos culturales que se ofrecen al turista residente. Es por lo tanto el resultado del total de experiencias turísticas y residenciales de los usuarios del sector, al que se añade los distintos mecanismos de producción de imagen del destino. Los mencionados problemas asociados a la masificación, mala planificación, carencias en infraestructuras y servicios, así como la destrucción de paisajes de alta calidad han generado el desprestigio de la imagen turística de determinados municipios (Castiñeira, Orozco y Vera, 1995). Ante esta situación, la respuesta de las empresas constructoras ligadas al sector turístico-residencial ha sido trasladar su actividad a zonas del interior sin desarrollo turístico. Este desplazamiento también se ha visto impulsado por otros factores tales como la búsqueda de suelo más barato y el agotamiento del suelo urbanizable en los municipios costeros

\subsubsection{Tipología constructiva}

Definición: características físicas de la vivienda: unifamiliar aislada, adosada, apartamento turístico, con/sin jardín, con/sin piscina, etc.

Contextualización: La tipología constructiva está en relación con la cercanía de la costa y el precio del suelo. En general, en primera línea de costa se construye en altura para maximizar la inversión del alto precio pagado por suelo, dado el valor añadido que da el paisaje costero. Conforme las promociones se alejan de la costa, disminuye la altura de los edificios, aumentando la horizontalidad del desarrollo urbano. Este modelo de expansión horizontal en el interior provoca altos consumos de suelo, y aumento de costes en la construcción de infraestructuras y servicios que los desarrollos urbanísticos llevan parejos (Vera, 2003). Por último cabe señalar que, al alejarse de la costa el proceso urbanizador, está apareciendo una nueva modalidad de urbanizaciones con campo de golf, el cual sustituye al mar como elemento que otorga valor añadido al producto inmobiliario (Espejo, 2004), tal y como está ocurriendo en la Región de Murcia. 


\subsubsection{Precios}

Definición: Nivel de precios. Valor económico de bienes (tales como suelo o vivienda) y servicios relacionados con el turismo residencial.

Contextualización: España era un país barato. La diferencia de renta entre España y los países más avanzados de la UE había sido una de los principales factores en la toma de decisiones de los turistas residentes para seleccionar las comunidades del Mediterréano español como la región donde comprar su segunda residencia (Abellán, 1993; Casado, 2000). No obstante, como consecuencia del fuerte crecimiento de la demanda de viviendas turísticas, los precios de la vivienda han aumentado notablemente (Martínez y Maza, 2003: 11$)^{8}$. Y como ha sido repetidamente señalado en los trabajos sobre los impactos del turismo (Torres, 2003), la llegada de turistas ha ocasionado un aumento en los precios del resto de artículos y productos consumidos por los turistas y residentes. En definitiva, está anulándose el factor precio como ventaja comparativa del turismo residencial frente a otros destinos competidores.

\subsubsection{Paisaje, recursos naturales y playas}

Definición: Paisaje, playas y recursos naturales. El entorno natural y recursos naturales propios de la provincia de Alicante y playas.

Contextualización: Como afirma Urry (1995), el consumo visual del paisaje es una de las principales actividades del turismo. En unos primeros momentos del turismo residencial en la Costa Blanca, este sector se benefició de unos paisajes muy poco explotados turísticamente (Gaviria, 1974). Posteriormente, la rápida urbanización del litoral, la nula planificación y la escasa valoración del medio ambiente en España ha ocasionado la destrucción de paisajes de gran calidad (Vera, 1999; OSE, 2006a). Este proceso de degradación paisajística ha ocasionado la hipervaloración por parte del mercado de aquellos espacios de calidad, cada vez más escasos y, por lo tanto, ha generado una mayor presión sobre el territorio (OSE, 2006b). Esta presión se ha visto facilitada por unas leyes urbanísticas que ponían escasos frenos a la expansión urbanística (European Parlament, 2005).

\subsubsection{Escena Urbana}

Definición: Las características del entorno o medio ambiente urbano, su estética, sus calles, tráfico, zonas verdes, elementos arquitectónicos, mobiliario urbano, paseos marítimos, etc.

Contextualización: Como señala H. García (2005), los subsectores principales de demanda del turismo residencial proceden de los compradores de segunda residencia españoles y del colectivo de jubilados de la Unión Europea asociados a los Movimientos Internacionales de Retirados (MIR). La propia naturaleza del turismo residencial ocasiona que, en este modelo turístico, la estancia sea más importante que el viaje. Así se explica la relevancia que posee la escena urbana en el sistema turístico residencial de la Costa Blanca. Los turistas residenciales hacen gran uso de los cascos urbanos de las localidades cercanas a su residencia. Los espacios urbanos se convierten lugares de uso y consumo turístico (Antón, 1998). No obstante, y a pesar de su importancia como producto turístico,

8 El precio de la vivienda se incrementó en un 15,89\% en el primer trimestre de 2006 según un informe del Colegio de Registradores de España (El País, 26-6-2006). 
los cascos históricos de los municipios de la Costa Blanca han padecido un acusado proceso de degradación (MUNRES, 1995) debido al rápido crecimiento de las ciudades costeras, a los problemas de planeamiento de las mismas y al escaso valor que hasta hace pocos años se le ha dado a los centros históricos en España (Ferrer, 2003).

\subsubsection{Inmigración de retirados}

Definición: Llegada de población extranjera retirada, mayoritariamente del norte y centro de Europa que, tras visitas turísticas anteriores, deciden asentarse en la provincia de Alicante.

Contextualización: La oferta turístico residencial de la Costa Blanca se ha convertido en un destino preferente para los jubilados extranjeros procedentes de la Unión Europea (Viruela y Domingo, 2001; Aledo, 2005). Según el Padrón Municipal de Habitantes, a principios del año 2006 había en la provincia de Alicante cerca de 100.965 ciudadanos mayores de 55 años procedentes de la Unión Europea, lo que supone el 20,3\% con respecto al total de la población de la provincia en ese rango de edad. Los contingentes más importantes en este rango de edad proceden de Reino Unido y Alemania. La compra de residencias por parte de este colectivo y su estancia ha generado importantes rentas en los municipios de acogida (Rodríguez y Warness, 2002). No obstante, su perfil sociodemográfico genera unas demandas de servicios que los municipios de acogida no están totalmente preparados para satisfacer (Casado, 1999).

\section{Análisis de las interrelaciones de las variables clave}

Una vez se ha identificado y contextualizado las variables clave, se está en disposición de profundizar en las interrelaciones que éstas mantienen con otros elementos del sistema. En este punto la metodología aquí propuesta se muestra especialmente útil, puesto que permite la aproximación a los posibles efectos que tendría en el conjunto del sistema la actuación sobre determinados elementos clave. Este paso metodológico proporciona información valiosa para orientar los procesos de toma de decisiones en materia turístico-residencial, identificando posibles relaciones causales entre los elementos que componen el sistema. De este modo, es posible averiguar qué elementos del sistema se verán modificados si se actúa sobre las ocho variables centrales.

Para llevar a cabo este paso se ha traspasado la matriz generada por el Panel de Expertos, con apoyo del programa MicMac, al programa Ucinet de análisis de redes. Este programa ofrece la posibilidad de generar una representación gráfica (Gráfico 2) de las interacciones de carácter fuerte ${ }^{9}$ existentes entre las ocho variables clave (en negro) y el resto de variables principales del sistema (en gris).

Este gráfico proporciona dos líneas de interpretación. En primer lugar, permite averiguar el modo en que se puede incidir en una de las variables centrales a través de los elementos que ejercen influencia sobre ella; y en segundo lugar, permite identificar los componentes del sistema que se verían afectados si se actuase sobre una de las variables centrales. Un ejemplo de la primera línea de interpretación propuesta sería el análisis de las influencias de otros elementos sobre la variable «Prestigio del destino». Habitualmente, las estrategias llevadas a cabo por el sector público y privado para la mejora de la imagen exterior de un destino se han centrado en el diseño e implementación de campañas de marketing y

9 Puntuadas con un 3 en la matriz MicMac. 


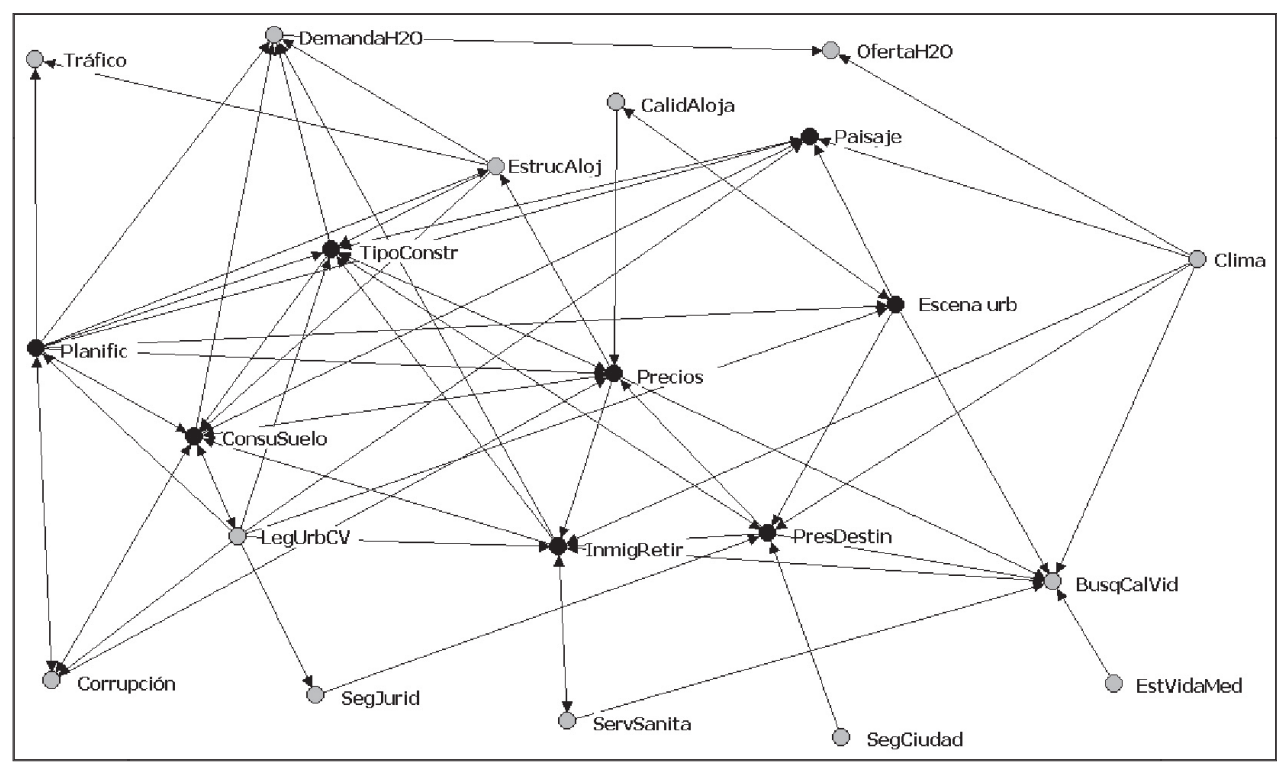

GráfICo 2. Relaciones de influencia entre las variables clave y el resto de variables principales del sistema.

Elaboración propia.

promoción del destino. Sin embargo, este análisis estructural nos indica que las variables que más determinan el prestigio del destino están relacionadas con las características de la escena urbana y su tipología constructiva, así como con los niveles de seguridad jurídica y ciudadana del lugar. Por lo tanto, a la hora de plantear estrategias de cualificación de un destino turístico-residencial, este gráfico indica que resulta más eficaz iniciar actuaciones en estas áreas antes de llevar a cabo costosas campañas publicitarias.

Con relación a la segunda línea de interpretación, este análisis facilita la previsión de las consecuencias que puede tener la opción por una determinada política o estrategia de desarrollo turístico-residencial sobre otras variables del sistema. Por ejemplo, en el caso de la variable «Tipología constructiva», observamos que ésta ejerce relaciones de influencia fuerte, no sólo sobre variables como la demanda de agua del destino turístico, sino sobre otras variables clave como «Consumo de Suelo», «Precio» o «Prestigio del Destino». En este sentido, el tomador de decisiones debe tener en cuenta que medidas referidas a la tipología constructiva terminarán ejerciendo efectos sobre elementos tan importantes del sistema como los que se acaban de mencionar.

\section{Conclusiones}

Las características y déficits de la planificación en municipios turístico-residenciales españoles, señalados al principio de este artículo, plantean la necesidad de alternativas metodológicas y analíticas para los procesos de toma de decisiones en este campo. Con esta motivación, se ha expuesto una propuesta metodológica que, a través de un enfoque sistémico-estructural, permite conocer de manera integral el conjunto de variables que conforman el sistema del turismo residencial en la Costa Blanca. Mediante la creación de 
un Panel de Expertos, esta metodología asegura la generación de información cualificada y multidisciplinar. Con el trabajo del Panel de Expertos, se identificaron y priorizaron los elementos componentes del sistema, para posteriormente analizar las interrelaciones que tales elementos mantienen entre sí. El conocimiento de las relaciones de dependencia/influencia entre las variables del sistema permitió identificar las que pueden considerarse variables clave. Estas variables clave señalan las áreas de actuación prioritaria a la hora de introducir cambios en el sistema turístico-residencial. Esta metodología sigue, por lo tanto, una secuencia lógica y clara para la organización y uso de la información recopilada.

La herramienta metodológica que se ha expuesto en este artículo plantea grandes ventajas para la planificación turístico-residencial, aunque también algunas limitaciones. En relación a las limitaciones de esta herramienta, debe señalarse que, dado el carácter cualitativo de la metodología, su eficacia depende en todo momento de la calidad de la identificación de los elementos que componen el sistema, y que es la base para el trabajo posterior. Por este motivo, es necesario prestar especial atención al desarrollo de esta primera fase.

Asimismo, y puesto que uno de los objetivos de esta propuesta es el de simplificar una realidad de carácter complejo, parte de la información inicial irá desapareciendo a lo largo del proceso, bien durante la priorización de los elementos, bien durante la identificación de variables clave.

En cuanto a sus ventajas, cabe señalar que la metodología propuesta es especialmente útil en su aplicación a los procesos de toma de decisiones en materia turístico-residencial por tres motivos principales. En primer lugar, esta metodología permite un mejor conocimiento de las interconexiones existentes entre los componentes del sistema turístico-residencial. Este conocimiento favorece, por un lado, la previsión de los posibles efectos que tendrían sobre el conjunto o parte del sistema las actuaciones realizadas sobre determinados elementos y, por otro lado, la identificación de vías indirectas de actuación sobre elementos concretos del sistema.

En segundo lugar, esta herramienta facilita la identificación de áreas prioritarias de actuación. Este paso es fundamental a la hora de distribuir recursos escasos para la planificación turístico-urbanística, así como para maximizar la potencialidad de las actuaciones.

Por último, esta herramienta ofrece una visión integral del sistema turístico-residencial como conjunto de elementos interrelacionados de manera compleja. Este enfoque integral se aleja de la habitual e ineficaz tendencia en el planeamiento español hacia la actuación de carácter sectorial y generalmente parcial.

\section{Referencias bibliográficas}

ABELLÁN, A. (1993): «La decisión de emigrar en las personas de edad» en Estudios Geográficos, $\mathrm{n}^{\circ} 210$, enero-marzo.

ALEDO, A. (2004): «Turismo inmobiliario y la fagocitación de la naturaleza» en Construçao do saber urbano ambiental: a caminho sa transdisciplinariedad. Londrinas-Paraná (Brasil): Ediciones Humanidades.

ALEDO, A. (2005): «Los otros inmigrantes: residentes europeos en el sudeste español», en J. Fernández Rufete y Modesto García (eds.) Movimientos migratorios europeos. Murcia: UCAM.

ALEDO, A.; GARCÍA, H. y ORTIZ, G. (2006): «Participación pública para la reorientación de municipios turístico-residenciales: procesos y dilemas». Comunicación presentada en VIII Jornadas de UCAM de Antropología Social y Cultural: Más allá del Turismo, la Migración y la Transnacionalidad. Murcia: Universidad Católica de Murcia.

ALEDO, A.; MAZÓN, T. y MANTECÓN, A. (2007): «La insostenibilidad del turismo residencial» en Lagunas D. (coord.) Antropología y turismo. Plaza y Valdés. México. 
ALMENAR, R.; BONO, E. Y GARCÍA, E. (2000): La sostenibilidad del desarrollo: el caso valenciano. Fundación Bancaixa y Universidad de Valencia. Valencia.

ANTÓN, S. (1998): «La urbanización turística: de la conquista del viaje a la reestructuración de la ciudad turística» en Documents d'anàlisi geogràfica, $\mathrm{N}^{\mathrm{o}} 32$, pp. 17-43.

ARROCHA, A. (2005): «La hacienda local en los destinos turísticos: el caso de Torrevieja», en Mazón T. y Aledo A. (Eds.) Turismo residencial y Cambio Social: nuevas perspectivas teóricas y empíricas. Editorial Aguaclara. Alicante.

BAUD-BOVY, M. Y LAWSON, F. (1998): Tourism \& recreation. Handbook of planning and design. Architectural Press. Oxford.

BOTE, V. (1998): «El desarrollo del turismo en España: cambio de rumbo y oportunidades científicas» en Revista Valenciana de Estudios Autonómicos. Págs. 29-43.

BOULLON, R. (1985): Planificación del espacio turístico. Trillas. México.

CASADO, M.A. (1999): «Efectos sociodemográficos asociados con el desarrollo de segundas residencias: el caso de Torrevieja (1986-1996)» en Alquibla, Revista de investigación del Bajo Segura. Vol. 5, pp. 457-473.

CASADO, M.A 2000: «De turistas a residentes: inmigrantes retirados europeos en Torrevieja, Costa Blanca», en II Cong. Inter. sobre la migración en España en el cambio de siglo. Madrid, 5-7 de octubre.

CASTIÑEIRA, C.J.; OROZCO, J.C. Y VERA, J.R. (1995): «Turismo y organización del territorio: desajustes de un modelo de implantación y nuevas estrategias» en Cuadernos de geografía, pp. 439-474.

CLARY, D. (1993) Le tourisme dans l'espace français. París: Masson

COSTA, A. Y JIMÉNEZ J.L. (1999): «Turismo y urbanismo» en Bayón F., 50 años del turismo español. Un análisis histórico y estructural. Centro de Estudios Ramón Areces. Madrid, pp. 465-486.

ESPEJO, C. (2004): «Campos de golf y medio ambiente: una interacción necesaria» en Cuadernos de Turismo, 14; pp. 67-111.

EUROPEAN PARLIAMENT (2005): Resolution on the alleged abuse of the Valencian Land Law or Ley Reguladora de la Actividad Urbanistica (LRAU - law on development activities) and its effect on European citizens (2004/2208(INI)). Strasbourg.

EXCELTUR, (2005): Impactos sobre el entorno, la economía y el empleo de los distintos modelos de desarrollo turístico del litoral mediterráneo español, baleares y canarias, Resumen ejecutivo. Madrid.

FERRER, M. (2003): Los centros históricos en España: teoría, estructura, cambio. Gobierno de Navarra. Dpto. de Medio Ambiente, Ordenación del Territorio y Vivienda. Navarra.

FERRER, R.; RIBERA, D. y REIG, A. (1997): Jubilados en la costa alicantina. Publicaciones de la Universidad de Alicante. Alicante.

FCAC (Fundación Cajas de Ahorro Confederadas) (2003): La convergencia real de España en el seno de la Unión Europea. Madrid.

GARCÍA, H. (2005): «Un acercamiento al concepto de Turismo Residencial» en Mazón, T. y Aledo, A., (Eds.) Turismo residencial y Cambio Social: nuevas perspectivas teóricas y empíricas. Editorial Aguaclara. Alicante.

GARCÍA, H. y RODES, J., (2004): «Análisis y comparativa de cinco municipios turísticos de la Costa Blanca», en A. Alvarez Sousa (coord.) Turismo, ocio y deporte. Universidad da Coruña. A Coruña.

GARCÍA, M. (2005): «Mis adorables vecinos (ciudadanos europeos de interior)», en T. Mazón y A. Aledo Turismo residencial y cambio social. CAM-UA. Alicante.

GARTNER, W.C. (1987): «Environmental impacts of recreational home development» en Annals of Tourism Research, $\mathrm{n}^{\circ} 14:$ pp.38-57.

GAVIRIA, M. (1974): España a go-gó: turismo charter y neocolonialismo del espacio. Ediciones Turner. Madrid.

GODET, M. (1991): Prospectiva y planificación estratégica. G. Editores. Barcelona.

GODET, M. (2000): La caja de herramientas de la prospectiva estratégica. Serpa. Paris.

GÓMEZ, A.; PRIETO, A.M. y STRANGELAND, P. (2003): «Urbanismo, corrupción y delincuencia organizada: un proyecto en la Costa del Sol», en Boletín Criminológico, ${ }^{\circ} 65$. 
GREENPEACE, (2006): Destrucción a toda costa: informe sobre la situación del litoral español. pp. 99-121.

GUNN, C. (1994): Tourism planning. Taylor \& Francis. Washington.

GUNN, C. (1997): Vationscape. Taylor \& Francis. Washington.

HALL, C. (2000): Tourism planning: policies, processes and relationships. Prentice Hall. Essex.

HALL, C.M. y MÜLLER, D.K. (2004): Tourism, Mobility and Second Homes: Between Elite Landscape and Common Group.(Clevedon: Channel View Books.

INSTITUTO NACIONAL DE ESTADÍSTICA (2006): Padrón municipal de habitantes.

IVARS, J. (2003): Planificación turística de los espacios regionales en España. Síntesis. Madrid.

LEIPER, N. (1995): Tourism Management. TAFE Publications. Collingwood.

MARTÍNEZ, J. Y MAZA, L.A. (2003): Análisis del precio de la vivienda en España Documento de Trabajo $n^{\circ}$ 0307. Banco de España.

MATHIESON, A. y WALL, G. (1992): Tourism: economic, physical and social impacts. Longman. Essex.

MAZÓN, T. (2006): «Inquiring into Residential Tourism: the Costa blanca case», Tourism and. Hospitality Planing \& Development, vol. 3, pp. 89-97

MAZÓN, T. y ALEDO A. (2005): Turismo residencial y cambio social. CAM-UA. Alicante.

MILL, R. y MORRISON, A. (1992): The tourist system. An introductory text. Prentice Hall, International Edition. Essex.

MONREAL, J. (2001): Un nuevo mercado turístico: jubilados europeos en la Región de Murcia. Universidad de Murcia. Murcia.

MUNRES. (1994): Programa de Revitalización de Municipios con Turismo Residencial. Instituto Universitario de Geografía y Diputación de Alicante. Alicante.

NAVALÓN, R. (1995): El planeamiento urbano y el turismo residencial en los municipios litorales de Alicante. Instituto de Cultura Juan Gil-Albert. Alicante.

OLCINA, J. y RICO, A. (2005): «Un crecimiento territorial con pies de barro», en J. Romero y M. Alberola (coords). Los límites del territorio. El País Valenciano en la encrucijada. Universidad de Valencia. Valencia.

O'REALLY, K. (1995): «A new trend in European migration: contemporary British migration to Fuengirola, Costa del Sol» en Geographical Viewpoint, 23.

OSE (Observatorio de la Sostenibilidad en España) (2006a): Cambios de ocupación del suelo en España. Mundi-Prensa Libros. Madrid.

OSE (2006b): Sostenibilidad en España 2006. Mundi-Prensa Libros. Madrid.

PEÑA, J.; BONET, A.; BELLOT, J.; SÁNCHEZ, J.R.; EISENHUTH, D.; HALLET, S. y ALEDO, A. (2007): «Driving forces of land-use change in a cultural landscape of Spain. A preliminary assessment of the human-mediated influences» en E. Koomen, J. Stillwell, A. Bakema, H.J. Scholten (eds.) Modelling Land-Use Change. Springer. Holanda.

PERLES, J. (2006): «Clusters, competitividad y desarrollo local en los destinos turísticos residenciales de la Comunidad Valenciana. El caso de Calpe» en López D. (ed). Turismo en los espacios litorales: sol, playa y turismo residencial. Univeristat Jaume I. Castellón, pp. 313-337.

PORTER, M. (1990): La ventaja competitiva de las naciones. Plaza y Janés.

Barcelona.

RODRÍGUEZ, V. y CASADO, M.A (2000). Residentes extranjeros de edad en España. II Congreso sobre Inmigración en España. Universidad de Comillas. Madrid.

RODRÍGUEZ, V. y WARNESS, T.(2002). «Los residentes europeos mayores en España: repercusiones socioeconómicas y territoriales», en El Campo de las ciencias y las artes, Servicio de Estudios BBVA, no 139 , pp. 123-148.

ROMERO, J.; MORALES, A.; SALOM, J. y VERA F. (coord.). (2003): La periferia emergente la Comunidad Valenciana en la Europa de las Regiones. Ariel. Barcelona.

ROMERO, J. y ALBEROLA, M. (coords). (2005): Los límites del territorio. El País Valenciano en la encrucijada. Universidad de Valencia. Valencia.

SALVÁ, P. (1998): «Los modelos de desarrollo turístico en el Mediterráneo», en Cuadernos de Turismo. $\mathrm{n}^{\circ}$ 2: 7-24. 
TORRES, E. (2003): «El turismo residenciado y sus efectos en los destinos turísticos», en Estudios Turísticos, $\mathrm{n}^{\circ} 155-156$, pp. 45-70.

URRY, J. (1995): Consuming places. Routledge. London.

VERA, J. (1987): Turismo y urbanización en el litoral alicantino. Instituto Juan Gil Albert. Alicante.

VERA, J. (1992): «Turismo y crisis agraria en el litoral alicantino», en F. Jurdao (ed.) Los mitos del turismo. Endimión. Madrid.

VERA, J. (1994): «El modelo turístico del Mediterráneo español: agotamiento y estrategias de reestructuración», en Papers de turismo. $\mathrm{n}^{\circ}$ 14-15, pp. 131-147.

VERA, J. (1999): «Turismo y medio ambiente» en Bayón F. 50 años del turismo español. Un análisis histórico y estructural . Centro de Estudios Ramón Areces, pp. 487-502. Madrid.

VERA, J. (2003): «Desarrollo turístico y procesos de reorganización territorial» en Morales A., Nuevas dinámicas de desarrollo y ejes de integración territorial : Campo de Cartagena y Bajo Segura. Ausur (Autopista del Sureste).

VERA, J. (2005): «Claves para la ordenación y gestión del litoral», en J. Romero y M. Alberola (coords). Los límites del territorio. El País Valenciano en la encrucijada. Universidad de Valencia. Valencia.

VERCHER, A. (2004): «La corrupción urbanística», en Claves, $\mathrm{n}^{\circ}$ 139, pp. 26-33.

VIRUELA, R. y DOMINGO, C. (2001): «Población extranjera en el País Valenciano», en Arxius, $\mathrm{n}^{\circ}$ 5, pp. 147-182. 